\title{
A SERIES TRANSFORMATION FORMULA AND RELATED POLYNOMIALS
}

\author{
KHRISTO N. BOYADZHIEV
}

Received 13 October 2004 and in revised form 18 April 2005

We present a formula that turns power series into series of functions. This formula serves two purposes: first, it helps to evaluate some power series in a closed form; second, it transforms certain power series into asymptotic series. For example, we find the asymptotic expansions for $\lambda>0$ of the incomplete gamma function $\gamma(\lambda, x)$ and of the Lerch transcendent $\Phi(x, s, \lambda)$. In one particular case, our formula reduces to a series transformation formula which appears in the works of Ramanujan and is related to the exponential (or Bell) polynomials. Another particular case, based on the geometric series, gives rise to a new class of polynomials called geometric polynomials.

\section{Introduction}

In this paper we present and discuss the following formula:

$$
\sum_{n=0}^{\infty} \frac{g^{(n)}(0)}{n !} f(n) x^{n}=\sum_{n=0}^{\infty} \frac{f^{(n)}(0)}{n !} \sum_{k=0}^{n}\left\{\begin{array}{l}
n \\
k
\end{array}\right\} x^{k} g^{(k)}(x)
$$

where $f, g$ are appropriate functions and $\left\{\begin{array}{l}n \\ k\end{array}\right\}$ are the Stirling numbers of second kind $\left(\left\{\begin{array}{l}n \\ k\end{array}\right\}\right.$ is the number of partitions of a set of $n$ elements into $k$ disjoint nonempty subsets, $[14,35])$.

An important feature of this formula is the fact that when $f$ is a polynomial, the righthand side is a finite sum and therefore (1.1) evaluates the left-hand side in a closed form. As shown in Section 5, formula (1.1) has also one other interesting feature: it transforms certain convergent series into asymptotic series. The main results in Section 5 include the asymptotic expansions in $\lambda$ of the incomplete gamma function $\gamma(\lambda, x)$ and the Lerch transcendent $\Phi(x, s, \lambda)$.

Sections 2 and 3 have a review character: we look at two special cases of (1.1) accompanied by historical notes. A sufficient condition for the validity of the formula is given in Section 4, where we prove (1.1) in a more general form, with the summation on the left-side going from $-\infty$ to $+\infty$. Finally, Section 6 contains some examples of series evaluation. 
3850 A series transformation formula and related polynomials

\section{Exponential polynomials}

If we set $g(x)=e^{x}$ in (1.1), the formula becomes

$$
\sum_{n=0}^{\infty} \frac{f(n)}{n !} x^{n}=e^{x} \sum_{n=0}^{\infty} \frac{f^{(n)}(0)}{n !} \phi_{n}(x),
$$

where

$$
\phi_{n}(x)=\sum_{k=0}^{n}\left\{\begin{array}{l}
n \\
k
\end{array}\right\} x^{k}
$$

are the exponential polynomials. We refer to (2.1) as the exponential transformation formula (ETF). An equivalent definition for the exponential polynomials is

$$
\phi_{n}(x)=e^{-x}(x D)^{n} e^{x}, \quad n=0,1, \ldots,
$$

with $(x D) f(x)=x f^{\prime}(x)$ (see (4.2) in Section 4). This equation can be written in the form

$$
\phi_{n}(x) e^{x}=(x D)^{n} e^{x}=\sum_{k=0}^{\infty} \frac{k^{n}}{k !} x^{k} .
$$

One has

$$
\begin{aligned}
& \phi_{0}(x)=1, \\
& \phi_{1}(x)=x, \\
& \phi_{2}(x)=x+x^{2}, \\
& \phi_{3}(x)=x+3 x^{2}+x^{3},
\end{aligned}
$$

and so forth. All coefficients $\left\{\begin{array}{l}n \\ k\end{array}\right\}$ are positive integers.

The polynomials $\phi_{n}$ form a basis in the linear space of all polynomials. Formula (2.2) shows how this basis is expressed in terms of the standard basis $1, x, x^{2}, \ldots, x^{n}, \ldots$ Solving for $x^{k}$ in (2.2) and writing the standard basis in terms of the exponential polynomials one finds that

$$
1=\phi_{0}, \quad x=\phi_{1}, \quad x^{2}=-\phi_{1}+\phi_{2}, \quad x^{3}=2 \phi_{1}-3 \phi_{2}+\phi_{3}, \quad \text { etc. }
$$

In general,

$$
x^{n}=\sum_{k=0}^{n}(-1)^{n-k}\left[\begin{array}{l}
n \\
k
\end{array}\right] \phi_{k},
$$

where $\left[\begin{array}{l}n \\ k\end{array}\right] \geq 0$ are the absolute Stirling numbers of the first kind. In particular

$$
\left[\begin{array}{c}
k \\
0
\end{array}\right]=0, \quad(k>0) ; \quad\left[\begin{array}{c}
k \\
1
\end{array}\right]=(k-1) ! ; \quad\left[\begin{array}{l}
k \\
k
\end{array}\right]=1 .
$$

More information on Stirling numbers can be found in $[8,14,20,34,35]$. 
Apparently, exponential polynomials were studied and used for the first time by Ramanujan in his notebooks [4, Chapter 3], but these results were not published in his lifetime. The first publications dealing with exponential polynomials in details originate from Bell $[2,3]\left(\phi_{n}\right.$ are also known as the single variable Bell polynomials) and Touchard $[27,28]$ (see the comments on [4, page 48] and also [8, page 133] and [24, 33]). There is a short note on these polynomials in Hardy's paper [15, 16, page 87]. The integers

$$
b_{n}=\phi_{n}(1)=\sum_{k=0}^{n}\left\{\begin{array}{l}
n \\
k
\end{array}\right\}
$$

are known as Bell or exponential numbers [2], [4, page 52], [10, 6.1], and [9, 22, 25, 31]. They give the number of ways a set of $n$ elements can be partitioned into nonempty disjoint subsets.

Formula (2.1) can also be found in the works of Ramanujan [4, page 58], who presented several interesting applications (see [4, Entry 10, its proof and the following examples, pages 57-65]).

It is easy to find the exponential generating function for the polynomials $\phi_{n}$; just set $f(x)=e^{x t}$ in (2.1) to get

$$
e^{x\left(e^{t}-1\right)}=\sum_{n=0}^{\infty} \phi_{n}(x) \frac{t^{n}}{n !} .
$$

The name "exponential polynomials" shows their close tie to the exponential function. A natural question is to find the ordinary generating function:

$$
h(x, t)=\sum_{n=0}^{\infty} \phi_{n}(x) t^{n} .
$$

This question is addressed in Section 5, see (5.2) and (5.15).

\section{Geometric polynomials and the geometric transformation formula}

We want to find now a transformation formula like (2.1), but without the factorials. For this purpose we choose for (1.1) the function

$$
g(x)=\frac{1}{1-x}, \quad(|x|<1),
$$

in which case $g^{(n)}(0) / n !=1$ (for all $\left.n\right)$. We get

$$
\sum_{k=0}^{\infty} f(k) x^{k}=\frac{1}{1-x} \sum_{n=0}^{\infty} \frac{f^{(n)}(0)}{n !} \omega_{n}\left(\frac{x}{1-x}\right),
$$

where $\omega_{n}$ are the polynomials

$$
\omega_{n}(x)=\sum_{k=0}^{n}\left\{\begin{array}{l}
n \\
k
\end{array}\right\} k ! x^{k}
$$


3852 A series transformation formula and related polynomials

with the inversion law [14, Problem 12, page 310]:

$$
x^{n}=\frac{1}{n !} \sum_{k=0}^{n}(-1)^{n-k}\left[\begin{array}{l}
n \\
k
\end{array}\right] \omega_{k}(x) \text {. }
$$

One has

$$
\omega_{0}(x)=1, \quad \omega_{1}(x)=x, \quad \omega_{2}(x)=2 x^{2}+x, \quad \omega_{3}(x)=6 x^{3}+6 x^{2}+x, \quad \text { etc. }
$$

The numbers

$$
\omega_{n}(1)=\sum_{k=0}^{n}\left\{\begin{array}{l}
n \\
k
\end{array}\right\} k !
$$

are known as the preferential arrangement numbers. Their combinatorial interpretation can be found in [9] and [22, 1.15].

Taking $f(x)=x^{m}, m=0,1,2, \ldots$ in (3.2) with the agreement $0^{0}=1$, one comes to the equation

$$
\sum_{k=0}^{\infty} k^{m} x^{k}=\frac{1}{1-x} \omega_{m}\left(\frac{x}{1-x}\right)
$$

analogous to (2.4). Keeping in mind that $(x D)^{m} x^{k}=k^{m} x^{k}$, we can view this as the rule

$$
(x D)^{m}\left\{\frac{1}{1-x}\right\}=\frac{1}{1-x} \omega_{m}\left(\frac{x}{1-x}\right), \quad m=0,1,2, \ldots,
$$

which explains the action of $(x D)^{m}$ on the rational function $1 /(1-x)$ in the same way (2.4) explains the action of this operator on $e^{x}$. We call the polynomials $\omega_{n}$ geometric polynomials, because their relation to the geometric series

$$
\sum_{k=0}^{\infty} x^{k}=\frac{1}{1-x}, \quad|x|<1,
$$

is similar to the relation of $\phi_{n}$ to the exponential series

$$
\sum_{k=0}^{\infty} \frac{x^{k}}{k !}=e^{x}
$$

Correspondingly, we call (3.2) the geometric transformation formula (GTF). The exponential and geometric polynomials are connected by the relation

$$
\omega_{n}(z)=\int_{0}^{\infty} \phi_{n}(z \lambda) e^{-\lambda} d \lambda
$$

which is verified immediately by using (2.2). One can derive now properties of $\omega_{n}$ from those of $\phi_{n}$. For instance, the exponential generating function for $\omega_{n}$ can be found by 
writing (2.10) in the form

$$
e^{x \lambda\left(e^{t}-1\right)}=\sum_{n=0}^{\infty} \phi_{n}(x \lambda) \frac{t^{n}}{n !},
$$

then multiplying both sides by $e^{-\lambda}$ and integrating for $\lambda$ from zero to infinity. In view of (3.11) this gives

$$
\int_{0}^{\infty} e^{-\lambda\left(1-x\left(e^{t}-1\right)\right)} d \lambda=\sum_{n=0}^{\infty} \omega_{n}(x) \frac{t^{n}}{n !},
$$

and therefore, the generating function for $\omega_{n}$ is

$$
\frac{1}{1-x\left(e^{t}-1\right)}=\sum_{n=0}^{\infty} \omega_{n}(x) \frac{t^{n}}{n !} .
$$

For every $x$, the left side in (3.14) is an analytic function of $t$ in some neighborhood of zero and the right side is its convergent Taylor series in this neighborhood. Note that (3.14) gives immediately $\omega_{n}(-1)=(-1)^{n}$.

A straightforward application of (3.7) follows from the observation that for every $|x|<$ 1 and every integer $m \geq 0$,

$$
\sum_{k=1}^{\infty}\left(1^{m}+2^{m}+\cdots+k^{m}\right) x^{k}=\frac{1}{1-x} \sum_{k=1}^{\infty} k^{m} x^{k} .
$$

(To prove this, multiply the left side by $1-x$ and simplify.) Therefore,

$$
\sum_{k=1}^{\infty}\left(1^{m}+2^{m}+\cdots+k^{m}\right) x^{k}=\frac{1}{(1-x)^{2}} \omega_{m}\left(\frac{x}{1-x}\right) .
$$

The summation of the series in (3.7) has a rich history, see [4, Chapter 5], [13, equation (4.10)], [14, equation (7.46), page 351], [19, 21], and [26, page 85]. There are numerous variations and extensions. The paper of Hsu and Shiue [17], for instance, provides some interesting examples and a good list of references.

The series in (3.7) can be summed also in terms of the Eulerian polynomials $A_{m}(x)$, [8, page 243] and [21,32]. Namely,

$$
\sum_{k=0}^{\infty} k^{m} x^{k}=\frac{1}{(1-x)^{m+1}} A_{m}(x) .
$$

Comparing this to (3.7) we find the relation between $A_{n}$ and $\omega_{n}$ :

$$
A_{n}(x)=(1-x)^{n} \omega_{n}\left(\frac{x}{1-x}\right)
$$


3854 A series transformation formula and related polynomials

Therefore, the GTF (3.2) can be written also in terms of the Eulerian polynomials:

$$
\sum_{k=0}^{\infty} f(k) x^{k}=\sum_{n=0}^{\infty} \frac{f^{(n)}(0)}{n !} \frac{A_{n}(x)}{(1-x)^{n+1}} .
$$

One has the representation

$$
A_{n}(x)=\sum_{k=0}^{n}\left\langle\begin{array}{l}
n \\
k
\end{array}\right\rangle x^{n-k}
$$

where $\left\langle\begin{array}{l}n \\ k\end{array}\right\rangle$ are the Eulerian numbers. A good reference for these numbers is [14]. (The Eulerian numbers $A(n, k)$ discussed in [8] differ slightly in the second index: $A(n, k)=$ $\left.\left\langle\begin{array}{c}n \\ k-1\end{array}\right\rangle.\right)$

Together (3.18) and (3.20) provide the following relation between Eulerian and Stirling numbers:

$$
\sum_{k=1}^{n}\left\langle\begin{array}{l}
n \\
k
\end{array}\right\rangle x^{n-k}=(1-x)^{n} \sum_{k=0}^{n}\left\{\begin{array}{l}
n \\
k
\end{array}\right\} k !\left(\frac{x}{1-x}\right)^{k} .
$$

(In particular, when $x=1 / 2$ this gives a solution to problem 11007 in the American Mathematical Monthly [36]).

We want to mention here also a recent paper by Wang and Hsu [30], who present one interesting Euler-Maclaurin type summation formula with a remainder, involving certain Eulerian fractions.

Throughout this paper, we prefer to use the geometric polynomials $\omega_{n}$ instead of the Eulerian polynomials, as $\omega_{n}$ participate in a more symmetric manner in most formulas and the Stirling numbers forming their coefficients appear to be more popular than the Eulerian numbers.

Formula (3.2) has a nice natural extension: taking

$$
g(x)=\sum_{k=0}^{\infty}\left(\begin{array}{c}
-r \\
k
\end{array}\right)(-1)^{k} x^{k}=\frac{1}{(1-x)^{r}}
$$

$(\operatorname{Re}(r)>0,|x|<1)$, one obtains from (1.1) the generalized GTF:

$$
\sum_{k=0}^{\infty}(-1)^{k}\left(\begin{array}{c}
-r \\
k
\end{array}\right) f(k) x^{k}=\frac{1}{(1-x)^{r}} \sum_{n=0}^{\infty} \frac{f^{(n)}(0)}{n !} \omega_{n, r}\left(\frac{x}{1-x}\right),
$$

where we call the polynomials

$$
\omega_{n, r}(z)=\frac{1}{\Gamma(r)} \sum_{k=0}^{n}\left\{\begin{array}{l}
n \\
k
\end{array}\right\} \Gamma(k+r) z^{k}
$$

the general geometric polynomials. Formula (3.11) extends to

$$
\omega_{n, r}(z)=\frac{1}{\Gamma(r)} \int_{0}^{\infty} \lambda^{r-1} \phi_{n}(z \lambda) e^{-\lambda} d \lambda
$$


and (3.8) extends in a symmetrical manner:

$$
(x D)^{m}\left\{\frac{1}{(1-x)^{r}}\right\}=\frac{1}{(1-x)^{r}} \omega_{m, r}\left(\frac{x}{1-x}\right), \quad m=0,1,2, \ldots
$$

When $f(z)=z^{m}$ in (3.23) one obtains also the following extension of (3.7):

$$
\sum_{k=0}^{\infty}(-1)^{k}\left(\begin{array}{c}
-r \\
k
\end{array}\right) k^{m} x^{k}=\frac{1}{(1-x)^{r}} \omega_{m, r}\left(\frac{x}{1-x}\right)
$$

or

$$
\sum_{k=0}^{\infty}\left(\begin{array}{c}
-r \\
k
\end{array}\right) k^{m} x^{k}=\frac{1}{(1+x)^{r}} \omega_{m, r}\left(\frac{-x}{1+x}\right)
$$

Now back to (3.2), setting there $x=-1$ and taking into account the interesting identity [14, Problem 6.76, page 317]

$$
\omega_{n}\left(\frac{-1}{2}\right)=\sum_{k=0}^{n}\left\{\begin{array}{l}
n \\
k
\end{array}\right\}(-1)^{k} \frac{k !}{2^{k}}=\frac{2}{n+1}\left(1-2^{n+1}\right) B_{n+1}
$$

( $B_{k}$ are the Bernoulli numbers), we come to the formula

$$
\sum_{k=0}^{\infty}(-1)^{k} f(k)=\sum_{n=0}^{\infty} \frac{f^{(n)}(0)}{(n+1) !}\left(1-2^{n+1}\right) B_{n+1}
$$

This formula can be used for evaluation of convergent alternating series or for Abel summation of divergent series. For instance, if $f(z)=z^{m}, m=0,1,2, \ldots$, we come to the Abel sum:

$$
\sum_{k=0}^{\infty}(-1)^{k} k^{m}=\frac{1-2^{m+1}}{m+1} B_{m+1}
$$

(counting $0^{0}=1$ ), which was essentially discovered by Euler (see [1, pages 1080-1081]).

\section{A sufficient condition for the validity of the transformation formula}

We will use again the equation

$$
(x D)^{m} x^{n}=n^{m} x^{n}
$$

true for all nonnegative integers $m$ and all $n$. We also need the representation

$$
(x D)^{m} g(x)=\sum_{k=0}^{m}\left\{\begin{array}{l}
m \\
k
\end{array}\right\} x^{k} g^{(k)}(x),
$$


3856 A series transformation formula and related polynomials

for any $m$-times differentiable function $g$ (see, e.g., [24, page 218]). This is easily proved by induction with the help of the relation

$$
\left\{\begin{array}{c}
m+1 \\
k
\end{array}\right\}=k\left\{\begin{array}{c}
m \\
k
\end{array}\right\}+\left\{\begin{array}{c}
m \\
k-1
\end{array}\right\}
$$

which follows immediately from the combinatorial interpretation of $\left\{\begin{array}{c}m \\ k\end{array}\right\}$ (see [14, page 266]).

The series transformation formula will be proved now in a slightly more general form than (1.1). Suppose we have two functions defined by power series

$$
f(x)=\sum_{n=0}^{\infty} a_{n} x^{n}, \quad g(x)=\sum_{n=-\infty}^{+\infty} c_{n} x^{n} .
$$

Theorem 4.1. Let $f(x)$ be an entire function and $g(x)$ be analytic on the annulus $K=$ $\{z, r<|z|<R\}$, where $0 \leq r<R$. If the series

$$
\sum_{n=-\infty}^{+\infty} c_{n} f(n) x^{n}
$$

converges absolutely on $K$, then

$$
\sum_{n=-\infty}^{+\infty} c_{n} f(n) x^{n}=\sum_{m=0}^{\infty} a_{m} \sum_{k=0}^{m}\left\{\begin{array}{c}
m \\
k
\end{array}\right\} x^{k} g^{(k)}(x)
$$

holds for all $x \in K$.

Proof. In view of (4.1), (4.2) can be written as

$$
\sum_{n=-\infty}^{+\infty} c_{n} n^{m} x^{n}=\sum_{k=0}^{m}\left\{\begin{array}{c}
m \\
k
\end{array}\right\} x^{k} g^{(k)}(x)
$$

for every integer $m \geq 0$. We multiply both sides by $a_{m}$ and sum for $m$ from zero to infinity

$$
\sum_{m=0}^{\infty} \sum_{n=-\infty}^{\infty} c_{n} a_{m} n^{m} x^{n}=\sum_{m=0}^{\infty} a_{m} \sum_{k=0}^{m}\left\{\begin{array}{c}
m \\
k
\end{array}\right\} x^{k} g^{(k)}(x) .
$$

Reversing the order of summation in the double series on the left gives (4.6). Changing the order of summation is legitimate, because (4.5) is absolutely convergent.

We want to point out that the condition on $f$ to be entire is crucial for the above proof (as we use its series (4.4) for $x=n$, that is,

$$
f(n)=\sum_{m=0}^{\infty} a_{m} n^{m}
$$

and the integer $n$ can be arbitrarily large). It becomes clear in the next section that this condition cannot be relaxed. 
Remark 4.2. Instead of the operator $(x D)$ we can use the more general operator $(x D+\lambda)$, $\lambda \in \mathbb{C}$, which has the property

$$
(x D+\lambda)^{m} x^{n}=(n+\lambda)^{m} x^{n},
$$

to prove in the same manner the more general formula

$$
\sum_{n=0}^{\infty} \frac{g^{(n)}(0)}{n !} f(n+\lambda) x^{n}=\sum_{n=0}^{\infty} \frac{f^{(n)}(0)}{n !} \sum_{m=0}^{n}\left(\begin{array}{c}
n \\
m
\end{array}\right) \lambda^{n-m} \sum_{k=0}^{m}\left\{\begin{array}{c}
m \\
k
\end{array}\right\} x^{k} g^{(k)}(x) .
$$

At the same time, replacing $f(x)$ by $f(x+\lambda)$ in (1.1) brings to the formula

$$
\sum_{n=0}^{\infty} \frac{g^{(n)}(0)}{n !} f(n+\lambda) x^{n}=\sum_{n=0}^{\infty} \frac{f^{(n)}(\lambda)}{n !} \sum_{k=0}^{n}\left\{\begin{array}{l}
n \\
k
\end{array}\right\} x^{k} g^{(k)}(x),
$$

with the same left-hand side.

Remark 4.3. With the notations (4.2) and (4.4), the series transformation formula can be written in the short form

$$
\sum_{n=-\infty}^{+\infty} c_{n} f(n) x^{n}=\sum_{m=0}^{\infty} a_{n}(x D)^{n} g(x)
$$

which is $f(x D) g(x)$ evaluated in two different ways.

The importance of the operator $(x D)$ for summation of series was understood by Schwatt, who used this operator in his book [26, Chapter 5]; see also the recent article by Knopf [19]. Interesting notes on transformation of formal series can be found in [8, page 221]. A formula similar to (3.2) with $f$ a polynomial and using finite differences instead of $(x D)$ was presented by Klippert in [18].

\section{Asymptotic series}

We have proved formula (1.1) under the condition that $f$ is entire. What if the function $f$ is not entire? Applying the ETF (2.1) to the analytic function

$$
f(z)=\frac{1}{z+\lambda}
$$

with a simple pole at $-\lambda$, we find for all $x$ and all $\lambda>0$ :

$$
\sum_{n=0}^{\infty} \frac{x^{n}}{n !(n+\lambda)}=e^{x} \sum_{n=0}^{\infty} \frac{(-1)^{n} \phi_{n}(x)}{\lambda^{n+1}} .
$$

Looking at this equation more carefully we notice that the first series, as a function of $\lambda$, is analytic with poles at $\lambda=-n, n=0,1, \ldots$. At the same time, the second series, if convergent for some $\lambda$, would represent an analytic function of $\lambda$ in a neighborhood of infinity, so the equality cannot hold. We will see, however, that (5.2) is in fact an asymptotic expansion. 
3858 A series transformation formula and related polynomials

A function $F(\lambda)$ has the asymptotic expansion (convergent or divergent) for $\lambda>0$ of the form

$$
F(\lambda) \sim \sum_{k=0}^{\infty} \frac{a_{k}}{\lambda^{k+s}}
$$

where $\operatorname{Re}(s) \geq 0$, if

$$
F(\lambda)=\sum_{k=0}^{n-1} \frac{a_{k}}{\lambda^{k+s}}+R_{n}, \quad \text { with }\left|R_{n}\right| \leq \frac{c_{n}}{\lambda^{n+s+1}}
$$

(cf. [23]). In order to prove that (5.2) is an asymptotic expansion, we first write the series on the left side in (5.2) as a Laplace integral:

$$
\sum_{n=0}^{\infty} \frac{x^{n}}{n !(n+\lambda)}=\sum_{n=0}^{\infty} \frac{x^{n}}{n !} \int_{0}^{\infty} e^{-n t} e^{-\lambda t} d t=\int_{0}^{\infty} e^{x e^{-t}} e^{-\lambda t} d t
$$

Next, by repeatedly applying $d / d t$ to the representation

$$
e^{x e^{t}}=\sum_{k=0}^{\infty} \frac{x^{k} e^{k t}}{k !}
$$

we obtain from (2.4) the following rule for differentiation of the iterated exponential:

$$
\left(\frac{d}{d t}\right)^{n} e^{x e^{t}}=\phi_{n}\left(x e^{t}\right) e^{x e^{t}}
$$

Taylor's formula centered at $t=0$ gives, for any $n \in \mathbb{N}$,

$$
e^{x e^{-t}}=\sum_{k=0}^{n} \frac{(-1)^{k} t^{k}}{k !} \phi_{k}(x) e^{x}+\frac{(-1)^{n+1} t^{n+1}}{(n+1) !} \phi_{n+1}\left(x e^{\theta}\right) e^{x e^{\theta}},
$$

where $\theta$ is between $-t$ and 0 and therefore $\theta \leq 0$ when $t \geq 0$. From here, with $\lambda>0$ :

$$
\int_{0}^{\infty} e^{x e^{-t}} e^{-\lambda t} d t=\sum_{k=0}^{n} \frac{(-1)^{k}}{\lambda^{k+1}} \phi_{k}(x) e^{x}+R_{n}(x, \lambda) .
$$

An estimate for the remainder $R_{n}(x, \lambda)$ now follows easily. Indeed, the polynomial $\phi_{n+1}$ has positive coefficients and we can write

$$
\left|\phi_{n+1}\left(x e^{\theta}\right) e^{x e^{\theta}}\right| \leq \phi_{n+1}(|x|) e^{|x|}
$$

(note that $e^{\theta} \leq 1$ ). This way

$$
\left|R_{n}(x, \lambda)\right| \leq \frac{\phi_{n+1}(|x|) e^{|x|}}{(n+1) !} \int_{0}^{\infty} t^{n+1} e^{-\lambda t} d t=\frac{\phi_{n+1}(|x|) e^{|x|}}{\lambda^{n+2}} .
$$


(If $x<0$, then $e^{x e^{\theta}} \leq 1$ and $e^{|x|}$ can be dropped.) We conclude that the integral in (5.5) is approximated by the finite sum

$$
\sum_{k=0}^{n} \frac{(-1)^{k}}{\lambda^{k+1}} \phi_{k}(x)
$$

with error $R_{n}(x, \lambda)$ such that for every $x$ and every $n \in \mathbb{N}$ one has, according to (5.11),

$$
R_{n}(x, \lambda) \longrightarrow 0, \quad \text { when } \lambda \longrightarrow \infty \text {. }
$$

The incomplete gamma function. The (lower) incomplete gamma function $\gamma(\lambda, x)(\operatorname{Re} \lambda>$ $0, x \geq 0$, see [23]) is defined by

$$
\gamma(\lambda, x)=\int_{0}^{x} u^{\lambda-1} e^{-u} d u
$$

Proposition 5.1. The following asymptotic expansion holds for $\lambda>0$ :

$$
\gamma(\lambda, x) \sim x^{\lambda} e^{-x} \sum_{n=0}^{\infty} \frac{(-1)^{n}}{\lambda^{n+1}} \phi_{n}(-x) .
$$

Proof. Setting $e^{-t}=u$ and changing $x$ to $-x$ we obtain from (5.5)

$$
\int_{0}^{\infty} e^{-x e^{-t}} e^{-\lambda t} d t=\int_{0}^{1} u^{\lambda-1} e^{-x u} d u=\sum_{n=0}^{\infty} \frac{(-1)^{n} x^{n}}{n !(n+\lambda)} .
$$

At the same time, the change of variable $u \rightarrow x u$ gives

$$
\gamma(\lambda, x)=\int_{0}^{x} u^{\lambda-1} e^{-u} d u=x^{\lambda} \int_{0}^{1} u^{\lambda-1} e^{-x u} d u
$$

and (5.15) follows from (5.2). The proposition is proved.

Our example naturally leads to a general theorem. First, we point out a simple and useful fact.

LEMma 5.2. For any function $g(z)$ analytic in a neighborhood of zero and for any nonnegative integer $n$ one has

$$
\left.\left(\frac{d}{d t}\right)^{n} g\left(x e^{t}\right)\right|_{t=0}=(x D)^{n} g(x)
$$

that is, the following Taylor expansion in the variable $t$ is true:

$$
g\left(x e^{t}\right)=\sum_{n=0}^{\infty} \frac{t^{n}}{n !}(x D)^{n} g(x)
$$

The proof requires just a simple computation and is left to the reader. 
3860 A series transformation formula and related polynomials

Theorem 5.3. Let $g(z)$ be analytic in a disk $K$ centered at the origin. Then for every $x \in K$ and $\operatorname{Re}(s)>0$, we have the asymptotic expansion in $\lambda>0$ :

$$
\sum_{n=0}^{\infty} \frac{g^{(n)}(0)}{n !} \frac{x^{n}}{(n+\lambda)^{s}} \sim \sum_{n=0}^{\infty}\left(\begin{array}{c}
-s \\
n
\end{array}\right) \frac{g_{n}(x)}{\lambda^{n+s}},
$$

where

$$
g_{n}(x)=(x D)^{n} g(x)=\sum_{k=0}^{n}\left\{\begin{array}{l}
n \\
k
\end{array}\right\} x^{k} g^{(k)}(x) .
$$

Further, if these functions have the property $\left|g_{k}(x)\right| \leq g_{k}(|x|)$, then the remainder has the estimate

$$
\left|R_{n}\right| \leq\left|\left(\begin{array}{c}
-s \\
n
\end{array}\right)\right| \frac{g_{n+1}(|x|)}{\lambda^{n+s+1}} .
$$

Proof. In order to strictly prove this asymptotic expansion we start by writing Taylor's formula of order $n \in \mathbb{N}$ for the function $g\left(x e^{-t}\right)$ of the variable $t$. We get, according to Lemma 5.2,

$$
g\left(x e^{-t}\right)=\sum_{k=0}^{n} \frac{(-1)^{k} t^{k}}{k !} g_{k}(x)+\frac{(-1)^{n+1} t^{n+1}}{(n+1) !} g_{n+1}\left(x e^{\theta}\right),
$$

where $\theta$ is between 0 and $-t$. At the same time we have

$$
g\left(x e^{-t}\right)=\sum_{n=0}^{\infty} \frac{g^{(n)}(0)}{n !} x^{n} e^{-n t},
$$

which is the Taylor series representation in the variable $x e^{-t}$. We multiply (5.23a) and (5.23b) by $t^{s-1} e^{-\lambda t} / \Gamma(s)$ and integrate for $t$ from zero to infinity. Using the representation

$$
\Gamma(s) a^{-s}=\int_{0}^{\infty} t^{s-1} e^{-a t} d t, \quad(a>0),
$$

we come to the left side in (5.20):

$$
\begin{aligned}
\frac{1}{\Gamma(s)} \int_{0}^{\infty} g\left(x e^{-t}\right) t^{s-1} e^{-\lambda t} d t & =\sum_{n=0}^{\infty} \frac{g^{(n)}(0) x^{n}}{n ! \Gamma(s)} \int_{0}^{\infty} e^{-n t} e^{-\lambda t} t^{s-1} d t \\
& =\sum_{n=0}^{\infty} \frac{g^{(n)}(0) x^{n}}{n !(n+\lambda)^{s}} .
\end{aligned}
$$

For the right-hand side we use again (5.24) and the identity

$$
\left(\begin{array}{c}
-s \\
k
\end{array}\right)=(-1)^{k} \frac{\Gamma(k+s)}{k ! \Gamma(s)} .
$$

The estimate for the remainder is immediate and the proof is complete. 
Remark 5.4. The representation (5.20) follows from the series transformation formula (1.1) formally applied to the function $f(z)=1 /(z+\lambda)^{s}$.

When $g(z)=e^{z}$, Theorem 5.3 gives

$$
\sum_{n=0}^{\infty} \frac{x^{n}}{n !(n+\lambda)^{s}} \sim e^{x} \sum_{n=0}^{\infty}\left(\begin{array}{c}
-s \\
n
\end{array}\right) \frac{1}{\lambda^{n+s}} \phi_{n}(x)
$$

and for $s=1$ we obtain (5.2).

(The function on the left side in (5.27) was studied by Hardy $[15,16]$, who obtained an asymptotic expansion in $x$. A general method for obtaining asymptotic expansions in $x$ of series like

$$
e^{-x} \sum_{n=0}^{\infty} \frac{f(n) x^{n}}{n !}
$$

is given by Ramanujan's Entry 10 on [4, page 57]. See also the notes on [4, page 64]).

Another interesting example is provided by the function $g(z)=1 /(1-z),|z|<1$. We use the differentiation rule

$$
\left(\frac{d}{d t}\right)^{n} \frac{1}{1-x e^{t}}=\frac{1}{1-x e^{t}} \omega_{n}\left(\frac{x e^{t}}{1-x e^{t}}\right)
$$

which follows from (3.8). Taylor's formula then gives the representation

$$
\frac{1}{1-x e^{-t}}=\sum_{k=0}^{n} \frac{(-1)^{k} t^{k}}{k !} \frac{1}{1-x} \omega_{k}\left(\frac{x}{1-x}\right)+\frac{(-1)^{n+1} t^{n+1}}{(n+1) !} \frac{1}{1-x e^{\theta}} \omega_{n+1}\left(\frac{x e^{\theta}}{1-x e^{\theta}}\right)
$$

with the property

$$
\left|\frac{1}{1-x} \omega_{k}\left(\frac{x}{1-x}\right)\right| \leq \frac{1}{1-|x|} \omega_{k}\left(\frac{|x|}{1-|x|}\right) .
$$

Therefore, when $\lambda>0,|x|<1$,

$$
\sum_{n=0}^{\infty} \frac{x^{n}}{(n+\lambda)^{s}} \sim \frac{1}{(1-x)} \sum_{n=0}^{\infty}\left(\begin{array}{c}
-s \\
n
\end{array}\right) \frac{1}{\lambda^{n+s}} \omega_{n}\left(\frac{x}{1-x}\right)
$$

with

$$
\left|R_{n}\right| \leq \frac{1}{\lambda^{n+s+1}}\left|\left(\begin{array}{c}
-s \\
n
\end{array}\right)\right| \frac{1}{(1-|x|)} \omega_{n+1}\left(\frac{|x|}{1-|x|}\right) .
$$


3862 A series transformation formula and related polynomials

The Lerch transcendent $\Phi(x, s, \lambda)$. This function (also known as the Lerch zeta function, or Hurwitz-Lerch zeta function) is defined for $\lambda>0$ and $|x| \leq 1, x \neq 1, \operatorname{Re}(s)>0$ or $x=1$, $\operatorname{Re}(s)>1$, by (see [11])

$$
\Phi(x, s, \lambda)=\sum_{n=0}^{\infty} \frac{x^{n}}{(n+\lambda)^{s}}
$$

When $|x|<1$, the series is convergent for all $s$. We have obtained the following.

COROLlary 5.5. For $|x|<1$ the Lerch transcendent has the asymptotic expansion (5.32) in $\lambda>0$.

For a different approach to this expansion see [12].

It is clear from the above considerations that the asymptotic expansion (5.32) still holds, when $|x| \leq 1, x \neq 1$, and $\operatorname{Re}(s)>0$, only without the given estimate for the remainder.

When $s=1$, (5.32) becomes

$$
\sum_{n=0}^{\infty} \frac{x^{n}}{n+\lambda} \sim \frac{1}{1-x} \sum_{n=0}^{\infty} \frac{(-1)^{n}}{\lambda^{n+1}} \omega_{n}\left(\frac{x}{1-x}\right)
$$

(cf. [37, Example 8.21, page 151]). For completeness, we list here also the expansion

$$
\sum_{n=0}^{\infty}(-1)^{n}\left(\begin{array}{c}
-r \\
n
\end{array}\right) \frac{x^{n}}{(n+\lambda)^{s}} \sim \frac{1}{(1-x)^{r}} \sum_{n=0}^{\infty}\left(\begin{array}{c}
-s \\
n
\end{array}\right) \frac{1}{\lambda^{n+s}} \omega_{n, r}\left(\frac{x}{1-x}\right)
$$

$(\operatorname{Re}(r)>0, \operatorname{Re}(s)>0,|x|<1)$ where the polynomials $\omega_{n, r}$ are defined in (3.24). This expansion follows from (5.20) with $g(z)=1 /(1-z)^{r}$.

Remark 5.6. As mentioned in Remark 4.3 at the end of Section 4, the series transformation formula (1.1) results from applying the operator $f(x D)$ to $g(x)$. When $f(x D)=$ $(x D+\lambda)^{-s}$, the action of this operator is described by (5.25), that is,

$$
(x D+\lambda)^{-s} g(x)=\frac{1}{\Gamma(s)} \int_{0}^{\infty} g\left(x e^{-t}\right) t^{s-1} e^{-\lambda t} d t=\sum_{n=0}^{\infty} \frac{g^{(n)}(0) x^{n}}{n !(n+\lambda)^{s}} .
$$

Formally, the asymptotic expansion (5.20) follows from the symbolic binomial series representation:

$$
(x D+\lambda)^{-s}=\frac{1}{\lambda^{s}}\left(1+\frac{x D}{\lambda}\right)^{-s}=\sum_{n=0}^{\infty}\left(\begin{array}{c}
-s \\
n
\end{array}\right) \frac{(x D)^{n}}{\lambda^{n+s}}
$$

applied to $g(x)$ with $(x D)^{n} g(x)=g_{n}(x)$.

An analytical theory of the operator $(x D+\lambda)^{r}$ was recently developed in a series of articles by Butzer et al. $[5,6,7]$. 


\section{Some examples of series evaluation}

As mentioned at the beginning, formula (1.1) evaluates the series on the left side in a closed form when $f$ is a polynomial. Let

$$
(z)_{m}=z(z+1) \cdots(z+m-1)
$$

be the rising factorial. Setting $f(z)=(z)_{m}$ and using the representation [14, page 263],

$$
(z)_{m}=\sum_{k=1}^{m}\left[\begin{array}{c}
m \\
k
\end{array}\right] z^{k}
$$

we obtain the following evaluations:

$$
\sum_{n=0}^{\infty}(n)_{m} \frac{x^{n}}{n !}=e^{x} \sum_{k=1}^{m}\left[\begin{array}{c}
m \\
k
\end{array}\right] \phi_{k}(x)
$$

for every $x$, according to the ETF (2.1) and

$$
\sum_{n=0}^{\infty}(n)_{m} x^{n}=\frac{1}{1-x} \sum_{k=1}^{m}\left[\begin{array}{c}
m \\
k
\end{array}\right] \omega_{k}\left(\frac{x}{1-x}\right)
$$

for every $|x|<1$, according to the GTF (3.2).

Next, for every polynomial $f$ of order $m$,

$$
f(z)=\sum_{n=0}^{m} a_{n} z^{n}
$$

and for every $p \in \mathbb{N}$, the GTF provides the evaluation

$$
\sum_{n=0}^{\infty}[f(n)]^{p} x^{n}=\frac{1}{1-x} \sum_{k=0}^{m p} \alpha_{k} \omega_{k}\left(\frac{x}{1-x}\right),
$$

where the coefficients $\alpha_{k}$ are given by

$$
\alpha_{k}=\sum_{j_{1}+j_{2}+\cdots+j_{p}=k} a_{j_{1}} a_{j_{2}} \cdots a_{j_{p}} .
$$

For example, using the representation

$$
\left(\begin{array}{c}
z \\
m
\end{array}\right)=\frac{1}{m !} \sum_{k=1}^{m}\left[\begin{array}{c}
m \\
k
\end{array}\right](-1)^{m-k} z^{k}
$$

[14, equation (6.13), page 263] one finds

$$
\sum_{n=m}^{\infty}\left(\begin{array}{c}
n \\
m
\end{array}\right)^{p} x^{n}=\frac{1}{1-x} \sum_{k=0}^{m p} \alpha_{k} \omega_{k}\left(\frac{x}{1-x}\right),
$$


3864 A series transformation formula and related polynomials

with

$$
\alpha_{k}=\frac{(-1)^{m p-k}}{(m !)^{p}} \sum_{j_{1}+j_{2}+\cdots+j_{p}=k}\left[\begin{array}{l}
m \\
j_{1}
\end{array}\right]\left[\begin{array}{l}
m \\
j_{2}
\end{array}\right] \cdots\left[\begin{array}{l}
m \\
j_{p}
\end{array}\right] .
$$

When $p=1$,

$$
\sum_{n=m}^{\infty}\left(\begin{array}{l}
n \\
m
\end{array}\right) x^{n}=\frac{1}{1-x} \sum_{k=0}^{m} \frac{1}{m !}\left[\begin{array}{c}
m \\
k
\end{array}\right](-1)^{m-k} \omega_{k}\left(\frac{x}{1-x}\right)=\frac{x^{m}}{(1-x)^{m+1}},
$$

according to (3.4). This can be verified independently by differentiating the geometric series (3.9) $m$-times and then multiplying the result by $x^{m} / m !$.

Another line of applications is described by the following example. Consider the multiple zeta function

$$
E_{m}(s)=\sum \frac{1}{\left(n_{1}+n_{2}+\cdots+n_{m+1}\right)^{s}},
$$

where $m \geq 1, s>m+1$, and $n_{1}, \ldots, n_{m}$ run from one to infinity, while $n_{m+1}$ runs from zero to infinity (we need this different range for $n_{m+1}$ in order to have the factor $\left(1-e^{-t}\right)^{-1}$ in the second integral below). We will evaluate $E_{m}(s)$ in terms of Riemann's zeta function:

$$
\zeta(s)=\sum_{n=1}^{\infty} n^{-s}, \quad \operatorname{Re}(s)>1,
$$

(cf. [29, page 499]). Using the representation (5.24) we write

$$
\begin{aligned}
\Gamma(s) E_{m}(s) & =\int_{0}^{\infty} t^{s-1} \sum e^{-\left(n_{1}+\cdots+n_{m+1}\right) t} d t=\int_{0}^{\infty} t^{s-1}\left(\frac{1}{1-e^{-t}}\right)\left(\frac{e^{-t}}{1-e^{-t}}\right)^{m} d t \\
& =\int_{0}^{\infty} t^{s-1}\left\{\left(\frac{1}{1-e^{-t}}\right) \sum_{k=1}^{m} M_{k}^{m} \omega_{k}\left(\frac{e^{-t}}{1-e^{-t}}\right)\right\} d t .
\end{aligned}
$$

Here we have used (3.4), setting for brevity

$$
M_{k}^{m}=\frac{(-1)^{m-k}}{m !}\left[\begin{array}{c}
m \\
k
\end{array}\right]
$$

(note that $M_{0}^{m}=0$ ). Consider now the polynomial $f(z)=\sum_{k=1}^{m} M_{k}^{m} z^{k}$. The GTF gives

$$
\begin{aligned}
\Gamma(s) E_{m}(s) & =\int_{0}^{\infty} t^{s-1}\left\{\sum_{n=1}^{\infty} f(n) e^{-n t}\right\} d t \\
& =\sum_{n=1}^{\infty} f(n) \int_{0}^{\infty} t^{s-1} e^{-n t} d t=\Gamma(s) \sum_{n=1}^{\infty} \frac{f(n)}{n^{s}}=\Gamma(s) \sum_{k=1}^{m} M_{k}^{m} \zeta(s-k) .
\end{aligned}
$$

Therefore,

$$
E_{m}(s)=\sum_{k=1}^{m} \frac{(-1)^{m-k}}{m !}\left[\begin{array}{c}
m \\
k
\end{array}\right] \zeta(s-k)
$$




\section{Acknowledgment}

The author wants to express his gratitude to the referees whose valuable remarks helped to improve the paper.

\section{References}

[1] R. Ayoub, Euler and the zeta function, Amer. Math. Monthly 81 (1974), no. 10, 1067-1086.

[2] E. T. Bell, Exponential numbers, Amer. Math. Monthly 41 (1934), 411-419.

[3] Exponential polynomials, Ann. of Math. (2) 35 (1934), no. 2, 258-277.

[4] B. C. Berndt, Ramanujan's Notebooks. Part I, Springer, New York, 1985.

[5] P. L. Butzer, A. A. Kilbas, and J. J. Trujillo, Compositions of Hadamard-type fractional integration operators and the semigroup property, J. Math. Anal. Appl. 269 (2002), no. 2, 387-400.

[6] , Fractional calculus in the Mellin setting and Hadamard-type fractional integrals, J. Math. Anal. Appl. 269 (2002), no. 1, 1-27.

[7] Mellin transform analysis and integration by parts for Hadamard-type fractional integrals, J. Math. Anal. Appl. 270 (2002), no. 1, 1-15.

[8] L. Comtet, Advanced Combinatorics, D. Reidel, Dordrecht, 1974.

[9] M. E. Dasef and S. M. Kautz, Some sums of some importance, College Math. J. 28 (1997), 52-55.

[10] N. G. de Bruijn, Asymptotic Methods in Analysis, Dover, New York, 1981.

[11] A. Erdélyi (ed.), Higher Transcendental Functions, vol. 1, McGrow-Hill, New York, 1955.

[12] C. Ferreira and J. L. López, Asymptotic expansions of the Hurwitz-Lerch zeta function, J. Math. Anal. Appl. 298 (2004), no. 1, 210-224.

[13] H. W. Gould, Euler's formula for nth differences of powers, Amer. Math. Monthly 85 (1978), no. $6,450-467$.

[14] R. L. Graham, D. E. Knuth, and O. Patashnik, Concrete Mathematics, Addison-Wesley, Massachusetts, 1994.

[15] G. H. Hardy, On the zeros of certain classes of integral Taylor series, Proc. London Math. Soc. (2) 2 (1905), 401-431, Part II.

[16] Collected Papers of G. H. Hardy (including joint papers with J. E. Littlewood and others). Vol. IV, Clarendon Press, Oxford, 1969.

[17] L. C. Hsu and P. J.-S. Shiue, On certain summation problems and generalizations of Eulerian polynomials and numbers, Discrete Math. 204 (1999), no. 1-3, 237-247.

[18] J. Klippert, Summing power series with polynomial coefficients, Amer. Math. Monthly 90 (1983), no. 4, 284-285.

[19] P. M. Knopf, The operator $\left(x \frac{d}{d x}\right)^{n}$ and its application to series, Math. Mag. 76 (2003), no. 5, 364-371.

[20] D. E. Knuth, Two notes on notation, Amer. Math. Monthly 99 (1992), no. 5, 403-422.

[21] H. K. Krishnapriyan, Eulerian polynomials and Faulhaber's result on sums of powers of integers, College Math. J. 26 (1995), no. 3, 119-123.

[22] L. Lovász, Combinatorial Problems and Exercises, 2nd ed., North-Holland, Amsterdam, 1993.

[23] F. W. J. Olver, Asymptotics and Special Functions, Academic Press, New York, 1974.

[24] J. Riordan, Combinatorial Identities, John Wiley \& Sons, New York, 1968.

[25] G.-C. Rota (ed.), Finite Operator Calculus, Academic Press, New York, 1975.

[26] I. J. Schwatt, An Introduction to the Operations with Series, Chelsea, New York, 1924.

[27] J. Touchard, Proprietes arithmetiques de certains nombres recurrents, Ann. Soc. Sci. Bruxelles A 53 (1933), 21-31 (French).

[28] Nombres exponentiels et nombres de Bernoulli, Canad. J. Math. 8 (1956), 305-320 (French). 


\section{A series transformation formula and related polynomials}

[29] I. Vardi, Determinants of Laplacians and multiple gamma functions, SIAM J. Math. Anal. 19 (1988), no. 2, 493-507.

[30] X. Wang and L. C. Hsu, A summation formula for power series using Eulerian fractions, Fibonacci Quart. 41 (2003), no. 1, 23-30.

[31] E. W. Weisstein, Bell Numbers, from MathWorld, http://mathworld.wolfram.com/BellNumber. html.

[32] _ Eulerian Numbers, from MathWorld, http://mathworld.wolfram.com/EulerianNumber.html.

[33] _ Exponential Polynomial, from MathWorld, http://mathworld.wolfram.com/ExponentialPolynomial.html.

[34] - Stirling Numbers of the First Kind, from MathWorld, http://mathworld.wolfram.com/ StirlingNumberoftheFirstKind.html.

[35] - Stirling Numbers of the Second Kind, from MathWorld, http://mathworld.wolfram. com/StirlingNumberoftheSecondKind.html.

[36] Western Maryland College Problems Group, Problem 11007, Amer. Math. Monthly 110 (2003), no. 4, 2 pp.

[37] E. T. Whittaker and G. N. Watson, A Course in Modern Analysis, 4th ed., Cambridge University Press, Cambridge, 1927.

Khristo N. Boyadzhiev: Department of Mathematics, Ohio Northern University, Ada, Ohio 45810, USA

E-mail address: k-boyadzhiev@onu.edu 


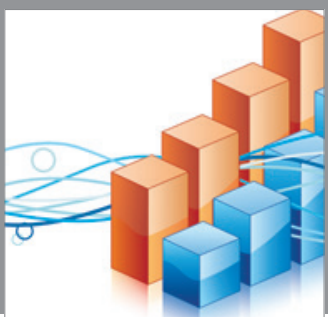

Advances in

Operations Research

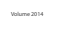

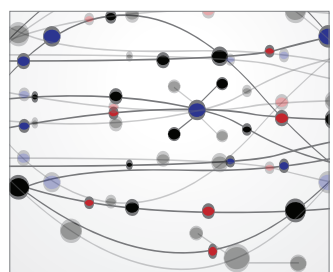

\section{The Scientific} World Journal
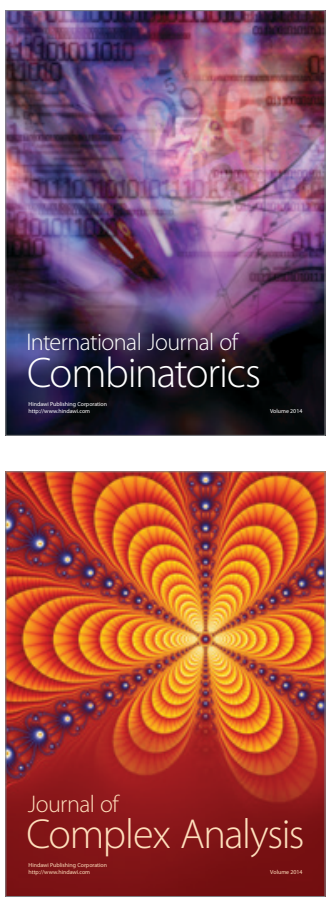

International Journal of

Mathematics and

Mathematical

Sciences
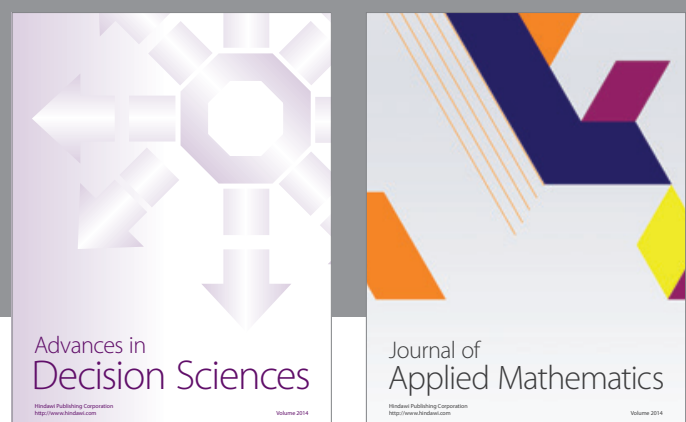

Journal of

Applied Mathematics
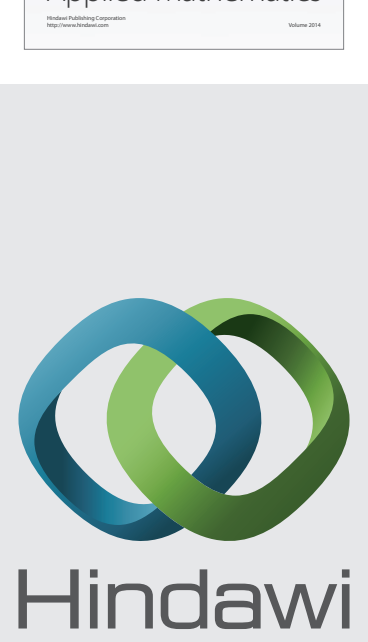

Submit your manuscripts at http://www.hindawi.com
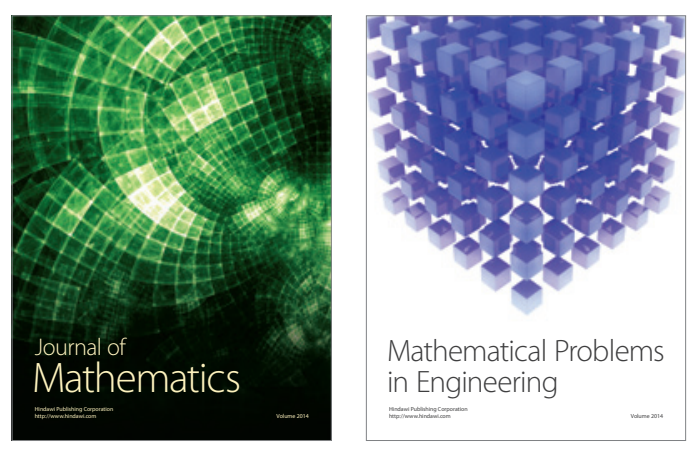

Mathematical Problems in Engineering
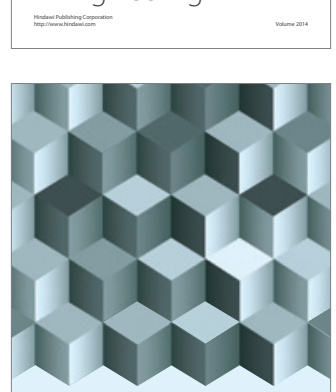

Journal of

Function Spaces
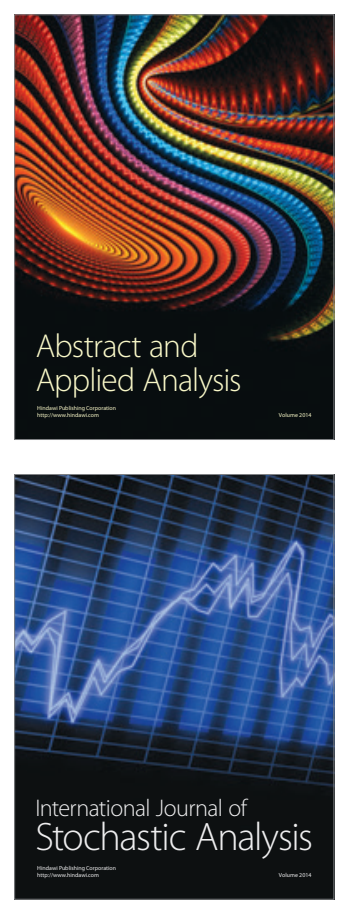

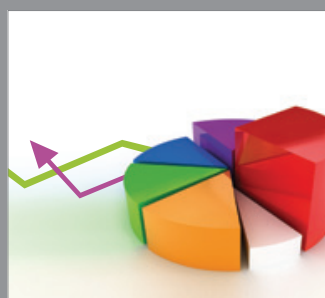

ournal of

Probability and Statistics

Promensencen
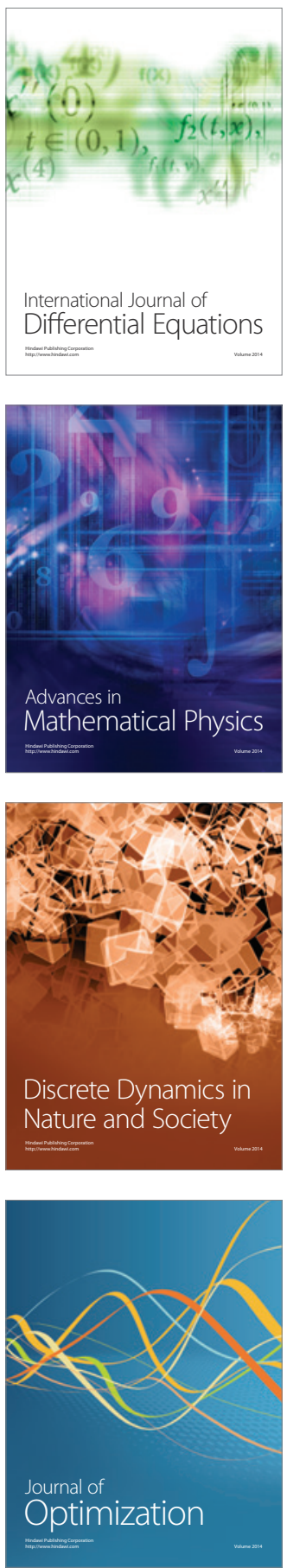